\title{
Original
}

\section{Análisis geográfico y temporal de la mortalidad evitable por cáncer cervicouterino en Costa Rica}

Amada Aparicio-Llanos, Melvin Morera-Salas

Resumen

Justificación y objetivo: El cáncer cervicouterino constituye una de las principales causas de muerte en las mujeres costarricense y más de la mitad de su ocurrencia tiene lugar antes de los 65 años de edad. El objetivo del presente estudio es identificar patrones de alta mortalidad evitable en las áreas de salud mediante un análisis temporal y espacial, con el fin de priorizar intervenciones desde los servicios de salud.

Métodos: Para analizar la evolución temporal se utilizó la tasa anual de mortalidad estandarizada y el porcentaje de cambio anual estimado mediante un modelo de regresión de Poisson. En el análisis espacial se utilizó la representación geográfica del índice de mortalidad estandarizada y de las áreas que registraron una mortalidad significativamente diferente al promedio nacional.

Resultados: Entre 1970 y 2005 la mortalidad evitable por cáncer cervicouterino decreció aproximadamente un $2 \%$. Se determinó la existencia de alta variabilidad entre áreas de salud (la tasa de mortalidad del área ubicada en el percentil 95 es 18 veces mayor que el área de salud ubicada en el percentil 5) y la sugerencia de un patrón con tasas de mortalidad superiores al promedio nacional en la zona atlántica y el sur del país.

Conclusión: Las decenas de muertes evitables anualmente y la alta variabilidad geográfica en las tasas de mortalidad entre áreas de salud por cáncer cervicouterino, son una alerta de potenciales problemas de atención de los servicios de salud. Se recomienda mejorar el proceso de detección temprana y abordaje oportuno del cáncer cervicouterino, priorizando intervenciones en las áreas de salud con altas tasas de mortalidad.

Centro Centroamericano de Población, Universidad de Costa Rica,

Caja Costarricense de Seguro Social.

Abreviaturas: CCSS, Caja Costarricense de Seguro Social; IME, índice de mortalidad estandarizada; TAE, tasa de mortalidad ajustada por edad Correspondencia: Amada Aparicio Llanos Apartado postal: 332-1000, San José

Correo electrónico: aaparici@ccss.sa.cr; aaparicio@fce.ucr.ac.cr

ISSN 0001-6002/2007/49/3/154-160 Acta Médica Costarricense, $\mathbb{O} 2007$ Colegio de Médicos y Cirujanos
Descriptores: Mortalidad evitable, análisis geográfico, cáncer cervicouterino

Key words: avoidable mortality, geographical analysis, cervicouterino Cancer

Recibido: 3 de enero de 2007

Aceptado: 24 de abril de 2007

El cáncer cervicouterino es un problema de salud pública en la mayoría de países del mundo y principalmente en las naciones en vías de desarrollo, ya que el $80 \%$ de las muertes por esa causa ocurre en países pobres. En Latinoamérica y el Caribe la incidencia llega al doble, y la mortalidad al triple de la que se presenta en los países desarrollados. ${ }^{1-2}$

En el período 2000-2005 los tumores malignos de útero constituyeron la tercera causa de muerte por cáncer en las mujeres costarricenses (tasa de 8.6/100 mil). La mitad de estas defunciones se produjeron entre los 15 y 64 años, y son consideradas como muertes evitables por existir intervenciones suficientemente efectivas para evitarlas.

El concepto de mortalidad evitable nace en 1976 con el nombre de "eventos centinela de salud" el cual evoluciona en 1983 a "evento evitable". ${ }^{4}$ La base de este concepto es la selección 
de causas de muerte en determinado grupo de edad, lo que se denomina en la bibliogafía como listas de mortalidad evitable. En 2006 existen 19 listas diferentes en el nivel internacional; ${ }^{5}$ la mayoría son adaptaciones de las elaboradas por Rutstein ${ }^{3}$ y Charlton. ${ }^{4}$ La más utilizada es la del Grupo de Servicios de Salud y Muertes Evitables de la Comunidad Europea. ${ }^{6}$

Una muerte se considera evitable cuando se puede prevenir mediante acciones individuales o sociales orientadas a controlar factores determinantes del entorno socioeconómico, o mediante la atención de la salud. ${ }^{7}$ La bibliografía divide las muertes evitables en prevenibles y tratables, según las características de intervención de salud a las que son susceptibles. ${ }^{8-9}$ Las causas prevenibles están más ligadas a algunos comportamientos y hábitos de vida, en los que la vía principal de intervención son las políticas de salud. Las causas tratables dependen mucho más de intervenciones realizables desde los servicios de salud. Desde este enfoque el indicador de mortalidad evitable ha sido utilizado como indicador de desempeño de los servicios de salud. ${ }^{10}$

El cáncer cervicouterino se incluye en el grupo de causas tratables donde las muertes pueden ser evitadas por un diagnóstico temprano y un tratamiento oportuno. No obstante, algunos autores ${ }^{11}$ clasifican esta neoplasia como $100 \%$ prevenible, dado que la lenta evolución de la enfermedad y la accesibilidad a las células de la zona de transición del cuello del útero permiten su estudio mediante la prueba del Papanicolaou, para detectar alteraciones citológicas premalignas, realizar análisis colposcópicos de confirmación diagnóstica y erradicar la enfermedad, si se trata a la paciente oportunamente.

La mortalidad por cáncer es uno de los temas de mayor aplicación del análisis geográfico, el cual ha tenido un gran avance en las últimas décadas gracias al acceso a las estadísticas de mortalidad y el desarrollo y disponibilidad de sistemas de información geográficos. ${ }^{12-13}$

Esta investigación realiza un análisis temporal con el fin de medir el comportamiento de las muertes por cáncer cervicouterino en Costa Rica en el periodo 1970-2005, y un análisis espacial de 2000 a 2005, con el objetivo de identificar diferencias regionales utilizando el enfoque de mortalidad evitable como indicador de potenciales problemas en la prestación en el nivel de áreas geográficas, que ayuden a priorizar intervenciones desde los servicios de salud.

\section{Materiales y métodos}

Los datos de mortalidad según grupo de edad y distrito se obtuvieron del Centro Centroamericano de Población y se agruparon para conformar las 103 áreas de salud establecidas para el primer nivel de atención de la CCSS, las cuales se constituyen en las áreas geográficas de estudio. Los datos de las poblaciones de 2000 a 2005, se obtuvieron de la Dirección Actuarial de la Caja Costarricense de Seguro Social (CCSS) $\mathrm{y}$ corresponden a proyecciones con base en el Censo de Población de 2000.

Las muertes evitables por cáncer cervicouterino se seleccionaron tomando como base la lista de mortalidad evitable propuesta por el grupo de trabajo de la Comunidad Europea sobre Servicios de Salud y Muertes Evitables, ${ }^{6}$ referida a las defunciones registradas con los códigos C53C55 del CIE-10, ${ }^{14}$ en los grupos de 15 a 64 años.

Para analizar la evolución temporal de las muertes en el periodo 1970-2005, se utilizó la tasa anual de mortalidad estandarizada por edad (TAE) y su porcentaje de cambio anual. La tasa de mortalidad fue ajustada por el método directo, empleando como población de referencia la registrada en el Censo de 2000.

$\mathrm{AE}=$ Proporción de la población estándar $* \sum \frac{\text { defunciones observadas }}{\text { personas }- \text { año en el grupo de edad }} * 100.000$

La tasa de cambio anual se estimó mediante regresión con un modelo aditivo de de Poisson, ${ }^{15-17}$ donde la variable dependiente son las defunciones, la variable de intervención es la población femenina entre los 15 y 64 años y las variables independientes son la edad en grupos decenales y el año de defunción.

En el análisis geográfico se utilizó el índice de mortalidad estandarizada (IME), método indirecto de ajuste de tasas que permite estimar el número de defunciones que se espera hubieran ocurrido en esos grupos, si los habitantes que los componen se murieran de acuerdo con la misma tasa de mortalidad de la población estándar, que en este caso es la población de Costa Rica del período 2000-2005.

IME $\frac{\text { defunciones observadas }}{\text { defunciones esperadas }} * 100$

Si el IME es igual a 100, la mortalidad del área de salud es igual al promedio nacional; si es superior a 100 el área de salud presenta un mayor riesgo de morir, y si es inferior a 100 el riesgo es menor respecto al promedio nacional.

La significancia estadística del IME se estimó usando una distribución de Poisson que es el modelo más recomendado en este caso, porque las muertes se producen como un evento discreto que toma pocos valores incluyendo el cero. ${ }^{17}$

La representación geográfica mediante mapas permite que se ubique visualmente cada área de salud en el territorio costarricense, por lo que en la Figura 1 se muestra a qué área pertenece cada espacio territorial para facilitar la interpretación de los posibles patrones de mortalidad evitable por cáncer cervicouterino, según las tasas de mortalidad ajustada por edad y los índices de mortalidad estandarizados. 


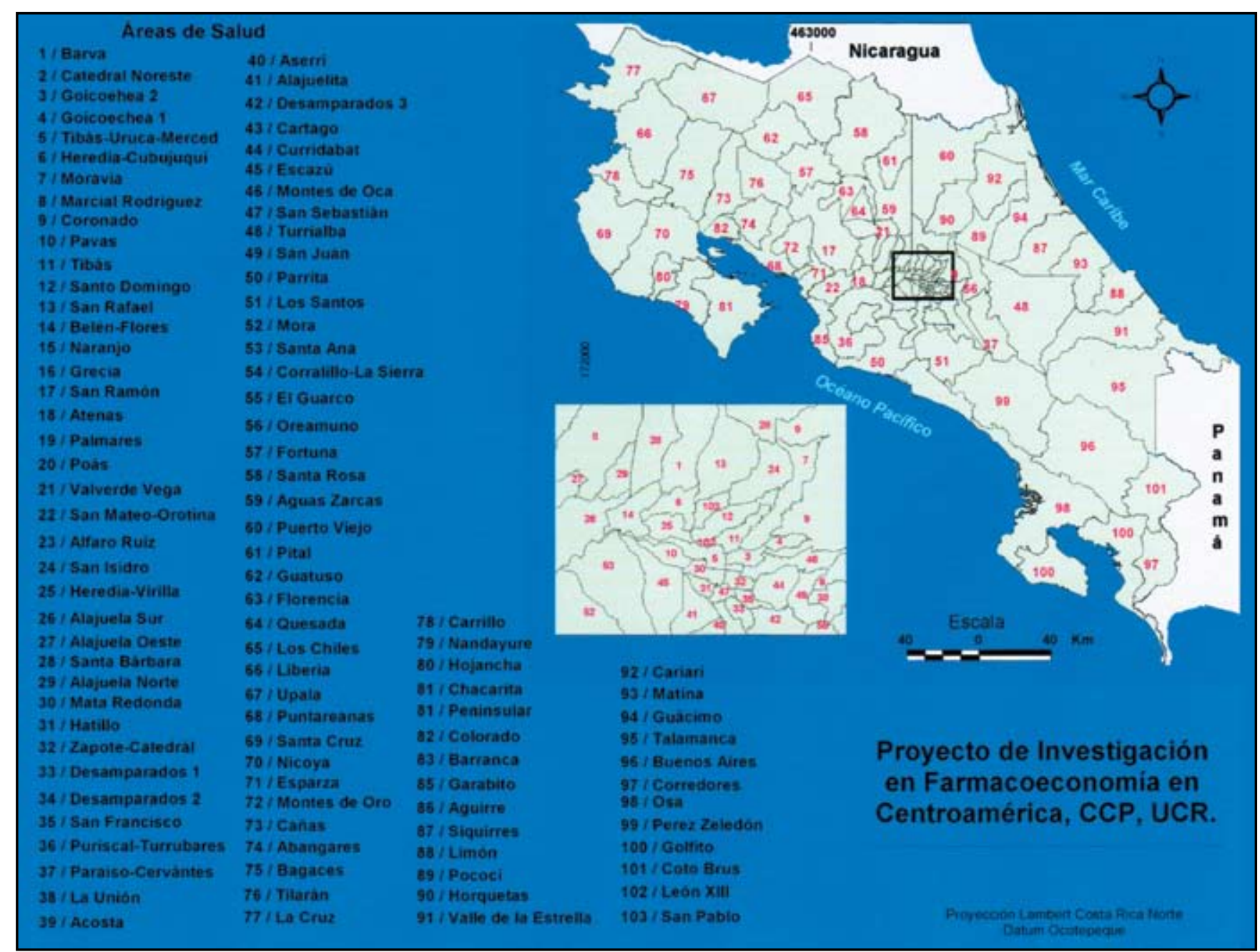

Figura 1. Representación geográfica de las áreas de salud.

En un primer mapa (Figura 3) se representó el valor de la tasa de mortalidad ajustada por edad, para lo cual se agruparon las áreas de salud en cuartiles y se representaron en una escala cromática de cuatro colores, que varía del celeste al azul intenso, donde los colores más claros corresponden a las áreas de salud con tasas relativamente más bajas y los más oscuros a las áreas de salud con tasas relativamente más altas.

En un segundo mapa (Figura 4) las áreas de salud se agruparon en tres categorías, según el valor del IME y su significancia estadística. A las áreas de salud que tienen un IME inferior al $100 \%$ y cuya diferencia es estadísticamente significativa $(\mathrm{p}<0.05)$ se les denominó "inframortalidad" $\mathrm{y}$ se colorearon con verde. A las áreas que presentan una mortalidad significativamente superior al promedio nacional (IME $>100 \%$ y p $<0.05$ ), se les llamó "sobremortalidad" y se colorearon con rojo. A las áreas cuyo IME no resultó significativamente diferente al $100 \%(p>=0.05)$ se les denominó "no significativas" y se colorearon con amarillo.

\begin{tabular}{|cccc|}
$\begin{array}{c}\text { Cuadro 1. Riesgo relativo de morir por cáncer } \\
\text { cervicouterino, según grupo de edad, } \\
\text { (estimación con la regresión de Poisson) }\end{array}$ \\
\hline Grupo edad & Riesgo relativo & Lím. inferior & Lím. superior \\
\hline $15-24$ & 1 & & \\
$25-34$ & 15.7 & 10.2 & 24.3 \\
$35-44$ & 56.3 & 36.8 & 86.0 \\
$45-54$ & 106.6 & 69.8 & 162.8 \\
$55-64$ & 177.9 & 116.6 & 271.5 \\
\hline
\end{tabular}

Los cálculos de las medidas de mortalidad y los indicadores de variabilidad geográfica se realizaron en Microsoft Excel y en el programa estadístico SPSS versión 10.0. En cuanto a la representación geográfica de la TAE y el IME se utilizaron los sistemas de información geográfica ArcView 1.3, con la georreferenciación del Proyecto de Investigación en Farmacoeconomía del Centro Centroamericano de Población de la Universidad de Costa Rica. 


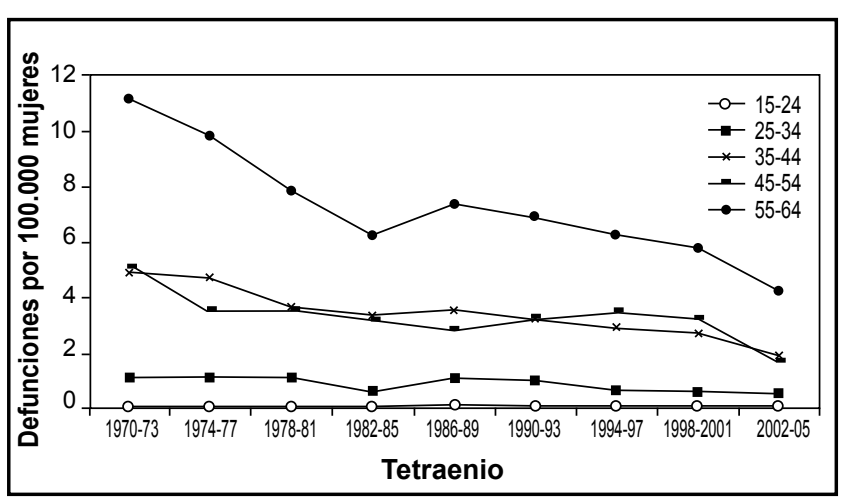

Figura 2. Tasas de mortalidad por cáncer cervicouterino, específicas por grupo de edad, Costa Rica, 1970-2005.

\section{Resultados}

Según los resultados de la regresión de Poisson, en el período 1970-2005 se registró un decrecimiento promedio anual en la tasa de mortalidad evitable por cáncer de útero del $2.2 \%$, con un intervalo de confianza del $95 \%$ de menos del $2.5 \%$ a menos del $1.9 \%$. También se determinó que el riesgo relativo de morir por cáncer de útero aumenta con la edad. En el Cuadro 1 se muestra el riesgo relativo por grupos decenales de edad, tomando como base el grupo de 15-24 años. La estimación indica, por ejemplo, que una mujer con edades entre 25-34 años tiene un riesgo 15.7 veces mayor de morir por cáncer de útero que una mujer entre 15-24 años. Este gradiente de mortalidad según edad se observa en la Figura 2, con excepción del grupo de 45-54 años, donde el gradiente se superpone al grupo anterior.

En el periodo 2000-2005 la tasa de mortalidad ajustada por edad, para cada área de salud, presenta una variación moderada, dado que el área de salud ubicada en el percentil 95 refirió una tasa de mortalidad 18 veces mayor que el área ubicada en el percentil 5 .

Se observa (Figura 3) que las tasas presentan un gran contraste visual, ya sea que estén en el primer o en el cuarto cuartil. No obstante, en algunas regiones hay uniformidad en la mortalidad, por lo que se emplea la misma intensidad de color, lo que sugiere posibles de patrones de mortalidad. Tal es el caso de las áreas de salud con alta mortalidad que encuentran en las regiones Pacífico Central, Pacífico Sur y Atlántica del país, lo que puede asociarse con un patrón geográfico costa- interior.

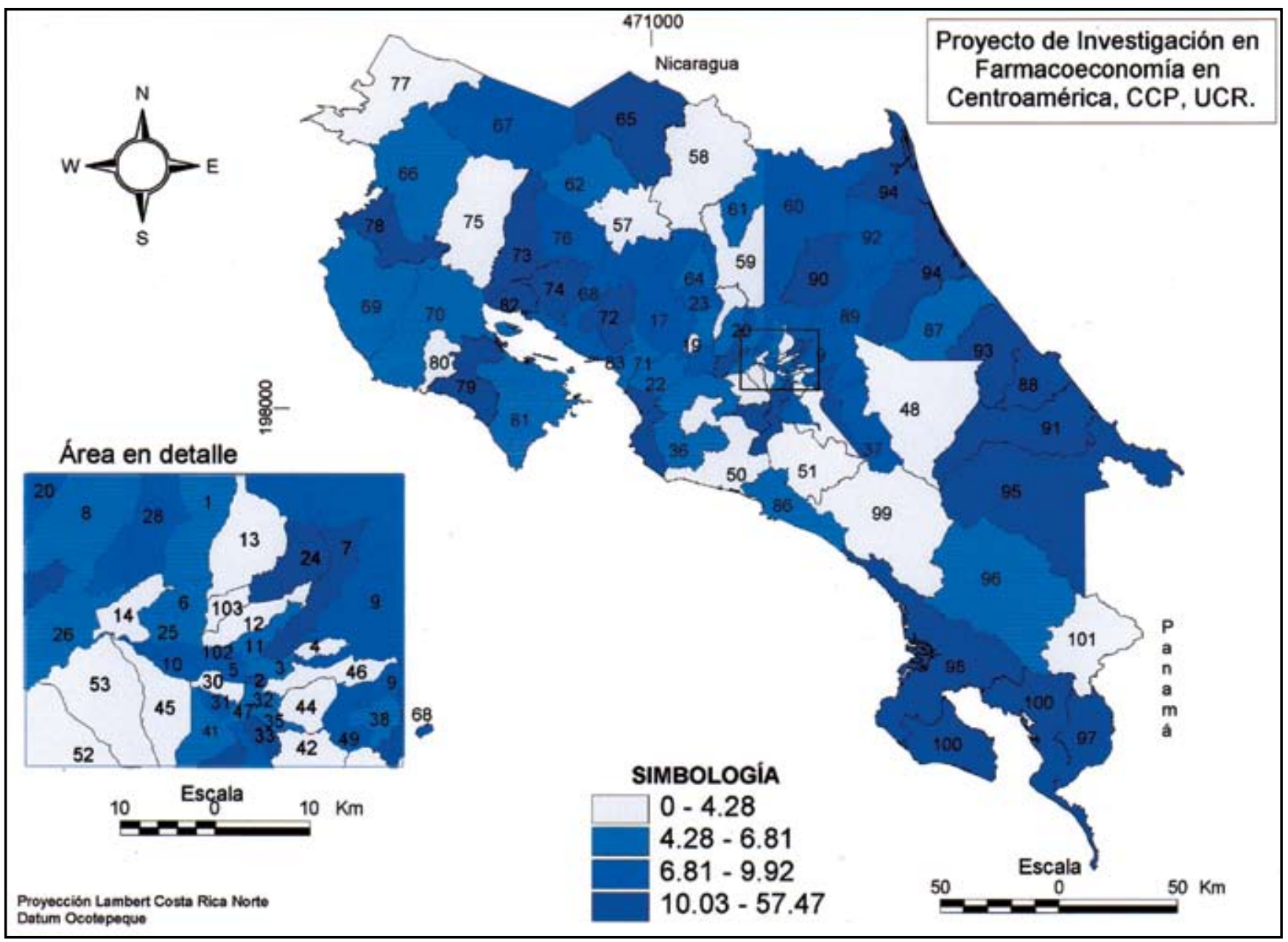

Figura 3. Distribución de la mortalidad por cáncer cervicouterino, Costa Rica, 2000-2005. 


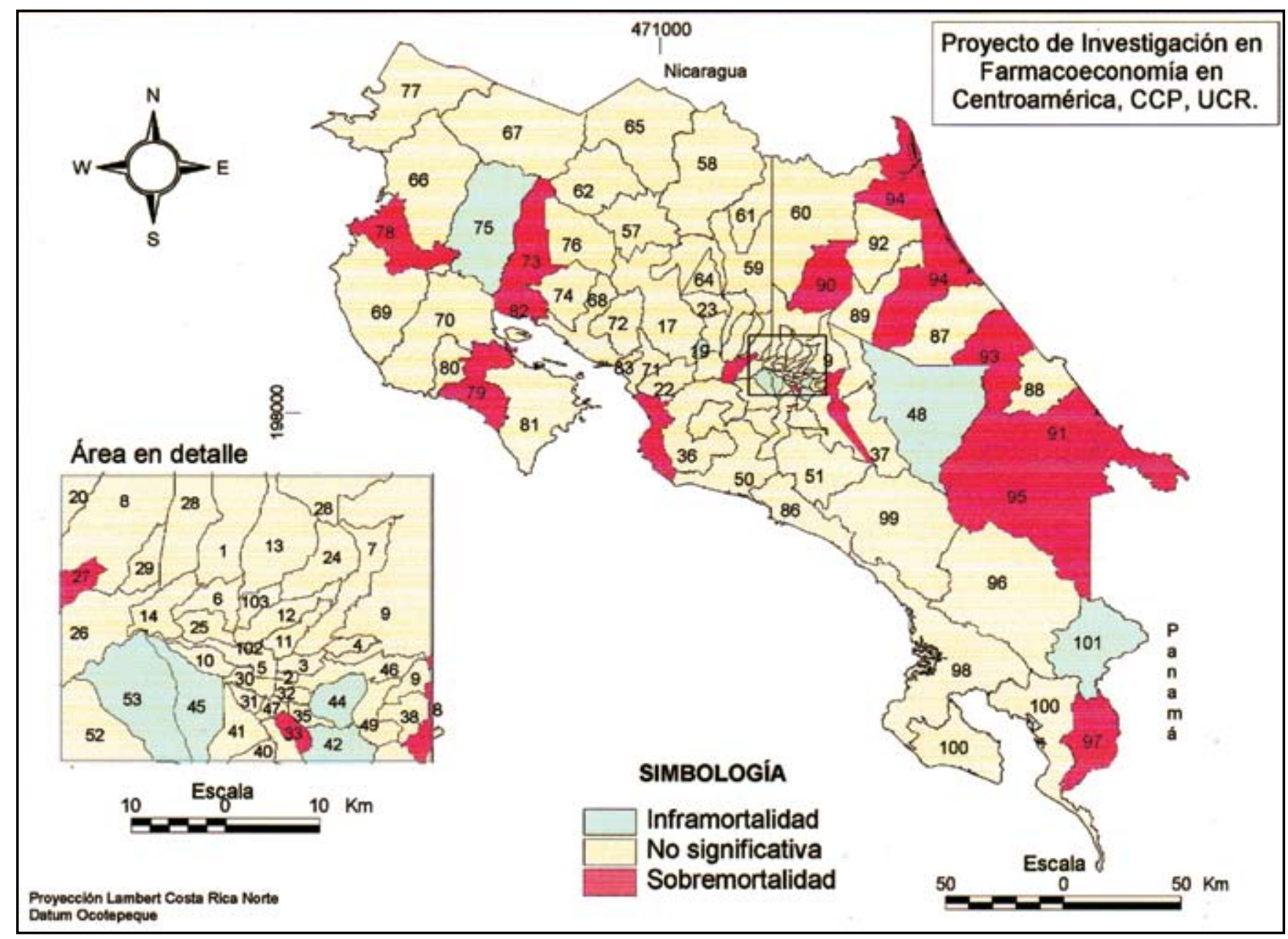

Figura 4. Áreas de Salud con mortalidad significativamente diferentes a la tasa nacional, Costa Rica, 2000-2005.

Con respecto al IME, las áreas de salud se distribuyeron de la siguiente manera: 13 áreas indicaron sobremortalidad; ocho, inframortalidad, y el resto no resultaron significativamente diferentes a la tasa nacional. Además, se observó un patrón geográfico acentuado solo en una parte de la zona Atlántica, donde las áreas de salud muestran sobremortalidad con respecto al comportamiento del país (IME $>100 \%, \mathrm{p}<0.05$ ).

\section{Discusión}

El descenso en las tasas de mortalidad evitable por cáncer de útero, de alrededor del $2.2 \%$, y su aceleración a partir de 1997 en todos lo grupos de edad, plantea la interrogante de cual es el peso que tiene una mejor intervención de los servicios de salud de la CCSS. Esta influencia ha sido medida en otros sistemas de salud, donde se demostró que siguiendo programas organizados de detección sistemática de lesiones preinvasoras y de estadios tempranos de aparición del carcinoma invasor de cuello de útero, se logra una reducción de la incidencia y mortalidad por esta patología. ${ }^{18-20}$
El análisis espacial de la mortalidad evitable por cáncer cervicouterino mostró alta variabilidad geográfica entre áreas de salud, una confluencia de áreas de salud con altas tasas de mortalidad en las zonas costeras (patrón-costa interior) y un patrón bien definido de sobremortalidad en la región Atlántica del país, donde un grupo de áreas de salud manifestó tasas de mortalidad evitable superiores al nivel nacional $y$ esas diferencias fueron estadísticamente significativas.

Esto implica que si se mejora el proceso de "detección temprana y abordaje oportuno del cáncer cervicouterino"18,21${ }^{23}$, sobre todo en las áreas de salud de la región Atlántica del país, frontera con Panamá y algunas zonas del Pacífico Central, donde se encontró un patrón geográfico de alta mortalidad evitable, se podría lograr disminuir la brecha entre áreas, medida con el indicador de mortalidad evitable por esta patología.

Otra intervención que ha cobrado importancia reciente es la propuesta de la Organización Mundial de la Salud para inmunizar contra el Virus del Papiloma Humano. ${ }^{24}$ Esta medida se está analizando actualmente en el Ministerio de Salud de Costa Rica, donde el tema central es el alto costo si se incluye en el esquema nacional de vacunación. 
En el planteamiento de estrategias de intervención también es importante determinar la influencia de factores individuales (edad, nacionalidad e ingreso, entre otros) y contextuales (disponibilidad de servicios de salud, diagnóstico precoz, existencia de ginecólogos y disponibilidad de equipo diagnóstico, entre otros) sobre la variabilidad en las tasas de mortalidad evitable entre áreas de salud.

Para que el indicador de mortalidad evitable sea más robusto y se pueda poder utilizar como indicador de desempeño de los servicios de salud, se debe ampliar el análisis de la mortalidad evitable a un número mayor de patologías y elaborar una lista de consenso, dadas las dificultades para seleccionar la serie más adecuada para el entorno costarricense, dentro de las múltiples listas utilizadas en el nivel internacional. ${ }^{5}$

La principal limitación de este estudio es la no inclusión de un ajuste para áreas pequeñas, lo que trae como consecuencia que los indicadores de mortalidad se vuelvan inestables en éstas. Se recomienda seguir las sugerencias de algunos autores acerca de suavizar las tasas ajustando los eventos de las áreas pequeñas con los de sus áreas vecinas, mediante un modelo jerárquico espacial. ${ }^{25-26}$

Agradecimiento: Este proyecto contó con el apoyo de una beca parcial sin condiciones del PPPI de la Merck Company Foundation, brazo filantrópico de Merck \& Co. Inc., Whitehouse Station, New Jersey, USA; a través del Proyecto de Investigación en Farmacoeconomía en Centroamérica del Centro Centroamericano de Población de la Universidad de Costa Rica.

\section{Abstract}

Justification and objective: Uterine cervical cancer is one of the main causes of death in Costa Rican women, more than half of those deaths occur before 65 years of age. The main objective of this investigation was to identify patterns of avoidable high mortality in different health areas following a temporo-spacial analysis in order to prioritize interventions right from the health services level.

Methods: To analyze the evolution we utilized the standardized annual mortality rate and the annual percentage of change estimated by a regression model according to Poisson.

For the geographical analysis we used the geographic representation of the standardized mortality ratio and the areas with a mortality rate significantly different from the national average.

Results: Between 1970 and 2005 avoidable mortality by uterine cervical cancer decreased approximately $2 \%$ and a mortality gradient was registered according to the age. With the geographical analysis we determined the existence of high variability between health areas (the mortality rate of the area located in percentile 95 is 18 times greater than the health area located in percentile 5) and a suggestion of a geographical pattern with rates of mortality superior to the national average both in the atlantic zone and the southern part of the country.

Conclusions: The annual occurrence of tens of avoidable deaths and the geographical variability in mortality rate between health areas, should constitute an alert sign of potential problems of attention regarding this entity. We recommended improving the detection process and the opportune approach to uterine cervical cancer, prioritizing interventions in the health areas with high mortality rates.

\section{Referencias}

1. Organización Mundial de la Salud. Datos sobre el cáncer. Nota descriptiva $\mathrm{N}^{\circ} 297$, febrero 2006. Disponible en: http://www.who.int/ mediacentre/factsheets/fs297/es/print.html

2. Situación de salud en las Américas: Indicadores básicos 2005. OPS, 2006. Disponible en: http://www.paho.org/spanish/dd/ais/IB-folleto2005.pdf.

3. Rutstein D, Berenberg W, Chalmers T, Child C, Fishman A, Perrin E. Measuring the quality of medical care: a clinical method. N Engl J Med 1976; 294: 582-588.

4. Charlton J, Hartley RM, Silver R, Holland WW. Geographical variation in mortality from conditions amenable to medical intervention in England and Wales. Lancet 1983; I: 691-696.

5. Gispert R, Barés A, Freitas A, Torné M, Puigdefäbregas A, Alberquilla A, et al. La mortalidad evitable: lista de consenso para la actualización del indicador en España. Gac. Sanit. 2006; 20: 184-193.

6. Holland W and the EC Working Group on Health Services and "Avoidable Death", eds. Eurpean Community Atlas of Avoidable Death 1985-1989. 3rd ed. Oxford: Oxford University Press; 1997.

7. Gispert R, Barés A, Freitas A, Torné M, Puigdefäbregas A, Alberquilla A, et al. Medida del resultado de las intervenciones sanitarias en España: una aproximación mediante el análisis temporal y espacial de la mortalidad evitable entre 1986-2001. Rev Esp Salud Pública 2006, 80: 139-155.

8. Treurniet F, Boshuizen C, Harteloh M. Avoidable mortality in Europe (1980-1997): a comparison of trends. J Epidemiol Community Health 2004; 58: 290-295.

9. James P, Doug M, Yang M. Avoidable mortality across Canada from 1975 to 1999. BMC Public Health 2006, 6: 137.

10. Nolte E, McKee M (2003). Measuring the health of nations: analysis of mortality amenable to health care. BMJ 2003; 327: 389-494.

11. Dzul K, Puerto M, González M. Cáncer cervicouterino: métodos actuales para su detección. Rev Biomed 2004; 15: 233-241.

12. Martínez Piedra R., et al. SIGEpi: Sistema de Información Geográfica en Epidemiología y Salud Pública. Boletín Epidemiológico/OPS, 22:4-5, 2001.

13. Organización Panamericana de la Salud (OPS). Cooperación técnica de la OPS en sistemas de información geográfica aplicados en epidemiología (SIG-EPI) en las Américas. Boletín Epidemiológico/ OPS, 17: 8-10, 1996.

14. Organización Panamericana de la Salud. Clasificación Estadística Internacional de Enfermedades y Problemas Relacionados con la Salud. 10a revisión. OPS, Washington, D.C., 1995.

15. Puig X, Ginebra J y Gispert R. Análisis de la evolución temporal de la mortalidad mediante modelos lineales generalizados. Gac Sanit. 2005; 19: 481-5.

16. Pennello GA, Devesa SS, Gail MH. Using a mixed model to estimate geographic variation in cancer rates. Biometrics 1999; 55: 774-81.

17. López-Abente G, Escolar A, Errezola M, Abraira V. Atlas de mortalidad por cáncer y otras causas en España 1978-1992. Fundación Científica de la Asociación Española contra el Cáncer Madrid, 1996. 
18. Sasiensi P, Adams J. Effect of screening on cervical cancer mortality in England and Wales. Analysis of trends with an age period cohort model. BMJ 1999; 318: 1244-5.

19. Parkin DM, Moss SM. An evaluation of screening policies for cervical cancer in England and Wales using a computer simulation model. J Epidemiol Community Healt 1986; 40: 143-153.

20. Herrero R, Brinton L, Reeves W, Brenes M, De Britton R, Gaitan E, Tinoco F. Screening for Cervical Cancer in Latin America: a casecontrol study. Int J Epidemiol 1992; 21: 1050-6.

21. Miller A. Report of a Worshop of the UICC project on evaluation of screening for cancer. International Journal of Cancer, 1990, 46: 76769.
22. Nazzal O, Reinero M, Abarzúa A, Liendo R y Palma C. Patología preinvasora del cerviz. Rev Chil Ginecol. 2003; 68: 189-196.

23. Novoa R. Análisis coste-efectividad del programa de detección sistemática del cáncer cervical en la región de Algarbe Portugal. Rev Esp Salud Pública 2004; 78: 341-353.

24. Organización Mundial de la Salud. Programas nacionales de control del cáncer: políticas y pautas para la gestión. OMS, Washington, D.C., 2004

25. Besag J, York JC, Mollié A. Bayesian image restoration, with two applications in spatial statistics (with discussion). Annals of the Institute of Statistical Mathematics. 1991. 43: 1-59.

26. Ministry of Health. Atlas of Cancer Mortality in New Zealand 1994 2000. Wellington: Ministry of Health, 2005.

\section{Costa Rica en el exterior}

\section{Ácido araquidónico del tejido adiposo y el síndrome metabólico en los adultos de Costa Rica.}

Clin Nutr 2007; mayo 2007

Williams ES, Baylin A, Campos H. Departamento de Nutrición, Escuela de Salud Pública de Harvard.

Justificación y objetivo: El ácido araquidónico, precursor de una serie de mediadores inflamatorios, puede contribuir al desarrollo de la resistencia de insulina. Examinamos la asociación entre el ácido araquidónico y el síndrome metabólico en Costa Rica, un país en el que el síndrome metabólico es altamente prevalente.

Métodos: Cada uno de los 484 participantes del estudio proporcionaron una muestra de sangre en ayunas y una biopsia del tejido adiposo que fue analizada para determinar la composición de ácidos grasos. Los criterios para el síndrome metabólico fueron los establecidos en el Tercer Informe del Equipo de Expertos Nacional del Programa de Educación en Colesterol. Los datos fueron analizados por regresión logística multivariable.

Resultados: Los sujetos con mayor grado de ácido araquidónico en el tejido adiposo tuvieron un riesgo mayor de sufrir síndrome metabólico a través de todos los quintiles: cociente de las probabilidades (OR, intervalo de confianza al 95\%) 1.00; 1.51 (0.78-2.91); 2.40 (1.26$4.55)$; 3.50 (1.84-6.66); y 6.01 (3.11-11.61); prueba para la tendencia, $\mathrm{P}<0.0001$, después del ajuste para la edad, el género y el área de la residencia. El ajuste adicional para los factores de riesgo metabólicos, incluyendo los ácidos grasos adiposos e índice de masa corporal, no modificó el resultado en forma significativa. El ácido araquidónico del tejido adiposo también se asoció independientemente a obesidad abdominal, hipertrigliceridemia, glucosa en ayuno elevada, y a presión arterial alta.

Conclusiones: Este estudio identificó al ácido araquidónico como marcador independiente importante de la desregulación metabólica. Una mejor comprensión del papel de este ácido graso en la patogenia del síndrome metabólico se justifica.

\section{Estimación de la carga del síndrome de la rubéola congénita en Costa Rica, 1996-2001.}

Pediatr Infect Dis J. 2007 May;26(5):382-6.

Jiménez G, Avila-Agüero ML, Morice A, Gutierrez H, Soriano A, Badilla X, Ref. S, Castillo-Solórzano C. Hospital Nacional de Niños, San José, Costa Rica.

Antecedentes: La epidemiología de la rubéola en Costa Rica cambió durante las décadas recientes, desplazándose los grupos susceptibles a aquellos de edad reproductiva. Este estudio estima la carga del síndrome de rubeola congénita (SRC) de 1996 al 2001 en este país.

Métodos: Tres métodos para calcular incidencia de SRC fueron utilizados. Se condujo una búsqueda retrospectiva ("casos observados") utilizando los registros de egreso hospitalario de niños nacidos entre 1996 y el 2001 con códigos seleccionados de CIE 9 y la CIE10 consistentes con SRC y los niños $<3$ meses de la edad con una prueba serológica positiva IgM anti-rubéola en el Hospital Nacional de Niños (HNN). Los casos fueron clasificados como sospechosos, compatibles o confirmados por SRC, e infección congénita por rubéola. La incidencia "esperada" de SRC fue calculada utilizando los casos reportados de rubéola (mujeres 15-45 años de edad) y las tasas de la fecundidad, asumiendo una probabilidad de SRC de 0,9 durante el primer trimestre del embarazo y 0,5 de casos asintomáticos de rubéola. Los casos "estimados" de SRC fueron calculados utilizando las tasas de incidencia reportadas de análisis de modelaje durante años epidémicos y endémicos. Resultados: de las 577 expedientes de egreso revisados y de los 66 niños reportados como anti-rubéola $\operatorname{IgM}(+), 40$ casos fueron considerados compatibles de SRC, 45 como confirmados, y 4 fueron los casos con infección congénita por rubéola identificados. El rango de la tasa de incidencia anual de SRC (por 1000 nacimientos vivos) fue como sigue: $=0.00-0.33$ "observado", $=0.00-0.35$ "esperado" $\mathrm{y}=0.5-1.5$ "estimado". Comparado con el número estimado de casos de SRC, solamente $27.2 \%$ de casos de SRC fueron detectados en la búsqueda retrospectiva y $10.1 \%$ se esperarían cuando se calcularon utilizando los casos reportados de rubéola. Conclusiones: La sub-detección de SRC utilizando los casos de rubéola reportados en mujeres en edad reproductiva y la búsqueda retrospectiva de SRC refuerza la importancia de sospechar SRC en la presencia de una manifestación única compatible. La confirmación por laboratorio es indispensable para implementar estrategias de eliminación de SRC y debería realizarse en todo caso sospechoso. 